\title{
La escalera del Real Colegio Seminario de Corpus Christi de Valencia (1599-1601): hipótesis de traza ${ }^{1}$
}

\section{The staircase of the Royal College of Corpus Christi Seminary of Valencia (1599-1601): tracing hypothesis}

\author{
$\underline{\text { R. Marín }}^{(*)}$, M. $^{\text {a }}$ C. López ${ }^{(*)}$
}

\section{RESUMEN}

Las escaleras claustrales con tramos volados representan la aportación más relevante del brillante episodio de la estereotomía valenciana de la Edad Moderna.

Este artículo muestra los resultados del análisis gráfico realizado a la escalera del Real Colegio de Corpus Christi de Valencia, su ejemplar más representativo, para determinar su posible traza. Con el apoyo de un levantamiento telemétrico mediante escáner láser 3D se ha constatado la existencia de un logrado método gráfico de diseño. El estudio ha considerado las operaciones que recomiendan los manuscritos de cantería y la tradición constructiva de sus predecesoras, las escaleras «de honor» de las casas nobles mediterráneas.

Esto ha permitido calibrar una estrategia de análisis útil para el estudio de otros ejemplos análogos; ponderar las informaciones ofrecidas por los tratadistas y confirmar la vigencia de los procedimientos tardomedievales de control de la forma mediante curvas directrices.

Palabras clave: Escalera; Edad Moderna; estereotomía; cantería; traza; montea; Historia de la Construcción.

\section{ABSTRACT}

The three-flight stairs with cantilevered sections show the most relevant contribution of the brilliant episode of the Valencian stereotomy of the Early Modern period.

This paper presents the results of the graphic analysis carried out on its most representative example to determine its possible tracing strategy, the staircase of the Corpus Christi Seminary of Valencia. With the support of a telemetric survey using a $3 D$ laser scanner, it has been verified the existence of an accomplished graphical method of design. The research has considered the operations recommended by stonecutting manuscripts and the constructive tradition of its predecessors, the «honour» staircases of the Mediterranean noble houses.

This has allowed us to calibrate a strategy of analysis useful for the study of other analogous examples; to consider the information offered by the treatise writers and the validity of Late Medieval methods of shape control using directional curves.

Keywords: Early Modern period; stereotomy; stonework; trace; template; History of Construction.

(*) Universitat Politècnica de València.

Persona de contacto/Corresponding author: rmarins@upv.es (R. Marín)

ORCID: http://orcid.org/oooo-0oo1-5311-5926 (R. Marín); http://orcid.org/oooo-ooo1-7542-3559 (M. ${ }^{a}$ C. López)

\footnotetext{
${ }^{1}$ Este artículo se inscribe en el proyecto de I+D «Geografías de la movilidad artística. Valencia en época Moderna» (HAR 2017-83070-P), financiado por el Ministerio de Economía, Industria y Competitividad del Gobierno de España.
}

Cómo citar este artículo/Citation: R. Marín, M. ${ }^{\text {a }}$ C. López. (2018). La escalera del Real Colegio Seminario de Corpus Christi de Valencia (1599-1601): hipótesis de traza. Informes de la Construcción, 70(550): e257. https://doi.org/10.3989/id.59131

Copyright: (C) 2018 CSIC. Este es un artículo de acceso abierto distribuido bajo los términos de la licencia de uso y distribución Creative Commons Reconocimiento 4.0 Internacional (CC BY 4.0). 
El Real Colegio Seminario de Corpus Christi (1586-1611), popularmente conocido como «El Patriarca» (1), es uno de los edificios más representativos del renacimiento valenciano. En su torre noreste acoge una excepcional «escalera claustral» (2) volada de caja abierta, resuelta en cantería, con sus tramos «corridos o continuados» y sus bóvedas «mezcladas por igual y por arista», en palabras de Joseph Gelabert ${ }^{2}$, tratadista mallorquín del siglo XVII (3). En fechas próximas a su construcción, Alonso de Vandelvira la denominó ${ }^{3}$ "escalera adulçada [adulçida] en cercha» (5 y 6) y, un siglo después, Juan de Portor y Castro, con mayor acierto, la describió como una «escalera capialzada engauchida por yladas en zircunferenzia» (7).

La confluencia de estos complejos recursos técnicos y su singular maestría convierten a dicho elemento, proyectado por Francisco Figuerola (8) entre 1599 y 1601, en uno de los ejemplos hispanos de tramos rectos más relevantes de la Edad Moderna $^{4}$. De hecho, esta variante «adulcida» podría haber surgido en tierras valencianas donde se conservan varios ejemplares, alguno muy temprano, destacando además la escalera del convento de Santo Domingo de Orihuela (15661568), atribuida a Joan Anglés y la escalera imperial del monasterio de San Miguel de los Reyes de Valencia (1600), de Joan Cambra.

En ninguna otra región se observa una conexión tan nítida con las propuestas tardomedievales de tramos rectos. Arturo Zaragozá (9) sostiene que los ejemplares andaluces y castellanos son posteriores y se ajustan a las trazas recogidas por Alonso de Vandelvira (1575-1591) en su célebre manuscrito, ampliamente difundido gracias a los trabajos de Barbé Coquelín de Lisle (10) y José Carlos Palacios (11). De hecho, la célebre escalera de la Real Chancillería de Granada (1578), citada por Vandelvira y estudiada por Macarena Salcedo (12), muestra cierta relación con el arquetipo del colegio oriolano, algo razonable dada la vinculación geográfica y artística de ambos territorios. Zaragozá, Calvo y Natividad (13) defienden incluso que sus homólogas francesas de caja también guardarían conexión con los modelos valencianos de patio, particularmente con la escalera del palacio de los Sancho en Onteniente (Valencia).

Esta valiosa contribución de la cantería levantina al moderno arte del corte de piedras cabe atribuirla a la existencia de un sustrato tecnológico previo capaz de alentar dichas innovaciones: pudo beneficiarse de los cambios introducidos en las escaleras «de honor» de las casas nobles mediterráneas, como ha señalado la profesora Gómez-Ferrer (14) y, acaso también, de las experiencias con superficies de doble curvatura incorporadas hacia 1450 por Francesc Baldomar al episodio local de las bóvedas aristadas (15).

\section{CONTEXTO DE LA INVESTIGACIÓN}

Los escritos de la época destacan la enorme dificultad que entraña el proyecto de estas escaleras de tramos rectos. Las disposiciones de 1565 del gremio de canteros de Valencia afirman que «solo los maestros las podían trazar» (16), una opinión compartida por Joseph Gelabert (1653) quien, después de alabar aquellas que "tenen engauxit» advierte que «sería home de molt gran art» quien consiga resolverlas (17). En la misma línea se manifiesta Alonso de Vandelvira, cuando advierte que son «las escaleras de más dificultad que las demás trazas» (18). Y también Thomas Vicente Tosca (1712) al indicar que «aunque sus cortes sean más dificultosos, es mucho mayor su magestad, y aun mayor la seguridad de su fábrica» (19).

Quizás por ello se concluyó hace tiempo su completa clasificación tipológica ${ }^{5}$ y se conoce a muchos de sus artífices, pero apenas han sido estudiadas con un enfoque técnico. Su pericia entraña un profundo conocimiento de la estereotomía que ha llevado décadas alcanzar para poder afrontar con garantías su análisis desde una doble perspectiva: la interpretación de los manuscritos y el examen de sus fábricas. Curiosamente, el mismo plazo transcurrido desde la pionera aproximación de José Carlos Palacios (1984) al estudio de los tres tipos descritos por Vandelvira.

Recientemente, Enrique Rabasa (22) ha explicado los trazados de las seis variantes de patio, más primitivas, del manuscrito de Gelabert y Rocío Carvajal (23) ha comparado las siete escaleras reseñadas en el manuscrito de Portor con las de otros textos de cantería. Estas aportaciones y el ya citado análisis de Macarena Salcedo sobre la escalera de la real Chancillería de Granada son los únicos estudios técnicos hasta la fecha.

En el episodio valenciano, además de los trabajos de Arturo Zaragozá y José Calvo, destacan las valiosas aportaciones de Mercedes Gómez-Ferrer ${ }^{6}$ (24) sobre la posible evolución técnica de las escaleras de las casas nobles valencianas y el léxico empleado en sus contratos. Esta investigadora ha descrito su eventual desarrollo, desde los modelos iniciales con bóvedas escarzanas que montaban unas sobre otras hasta la introducción y complicación del «capialçat» (capialzado) que llevó a experimentar soluciones más armoniosas de unión entre los tramos.

\footnotetext{
${ }^{2}$ Joseph Gelabert (1653) usa estos términos, que vienen de antiguo, en las trazas de sus escaleras. En el siglo XIX, el tratadista valenciano Manuel Fornés y Gurrea (4), aplicó idéntica terminología a sus propuestas tabicadas.

${ }^{3}$ La ortografía de las dos copias de este manuscrito es fluctuante. El título contiene el término «adulçada», pero es más frecuente «adulçida», del francés «adoucir».

${ }^{4}$ Los tratados españoles distinguen claramente entre los «caracoles» medievales y las «escaleras» modernas (de tramos rectos). El uso y desarrollo tecnológico de los primeros se circunscribe a la Edad Media y no cabe establecer paralelismos técnicos con las segundas. El ejemplo estudiado pertenece al grupo de las «escaleras», introducidas en los umbrales de la Edad Moderna, que combinan varios tramos rectos sostenidos por bóvedas. Su evolución busca resolver armónicamente los encuentros, facilitar la talla y reducir sus empujes. Las escaleras «claustrales» tienen, generalmente, tres tramos dispuestos en «C», predominando la variante «adulcida».

${ }^{5}$ Los primeros trabajos sobre el contexto peninsular iniciados por Harold Edwin Wethey (20) han sido ampliados recientemente por Jorge Martínez Montero (21).

${ }^{6}$ Gómez-Ferrer esboza la evolución de las escaleras de patio a partir de la distribución cronológica de los ejemplos conservados y algunas referencias documentales de otros desaparecidos.
} 

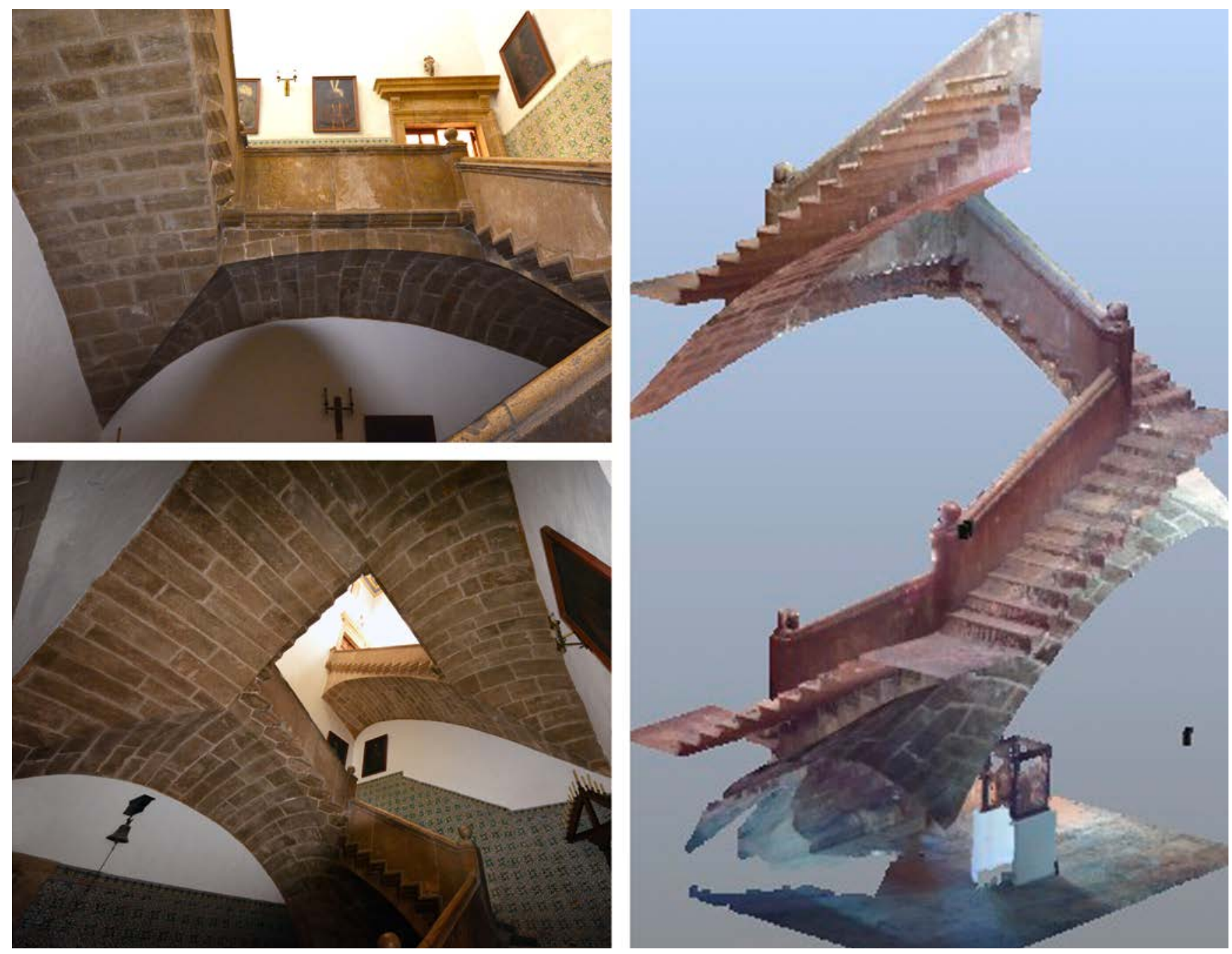

Figura 1. Vistas del intradós abovedado de la escalera (izquierda). Imagen digital 3D que muestra el intradós y trasdós de la escalera, sin los muros de cierre. Permite apreciar mejor el derrame de los capialzados de las bóvedas (derecha).

\section{RASGOS DISTINTIVOS DE LA ESCALERA}

La escalera del Colegio de Corpus Christi, está emplazada en una torre de planta casi cuadrada 7 de 7,82m x 7,42m (unos 26 x 24,5 pies valencianos de 0,302 m). Queda delimitada por gruesos muros de tapia valenciana de 21,2 metros de altura y 0,90 metros de grosor (23,5 x 1 varas), siguiendo la costumbre del momento en Valencia de integrar algunos elementos arquitectónicos de cantería (generalmente, portadas, escaleras y arquerías) en las obras con predominio de la albañilería.

Está compuesta por seis tramos (lo habitual son tres), cinco de ellos volados, construidos en dos fases. Los cinco primeros fueron proyectados por Figuerola entre 1598 y 1601. El últi- mo fue añadido en 1602 por Joan Baixet, Joan María y Bartolomé Abril (25) para dar acceso a la biblioteca. Todos ellos evolucionan con un sentido levógiro, alternando las uniones en arista y continuas de sus bóvedas bajo los rellanos, sin apoyo estructural alguno por su contorno interior (Figura 1).

La escalera arranca de una base maciza. El segundo tramo apoya en una bóveda «capialzada ${ }^{8} \mathrm{y}$ «engauchida $»^{9}$ con sus juntas transversales rectas y un pronunciado capialzo o derrame. El encuentro de este tramo con el siguiente configura una poderosa arista que da estabilidad a la estructura y evoca los enjarjes aristados de Francesc Baldomar. La tercera y cuarta tiradas descansan también sobre bóvedas capialzadas, pero aquí su derrame es un arco de

\footnotetext{
${ }^{7}$ Esta escalera parece haber sido proyectada con un criterio métrico similar al propuesto por Vandelvira y Portor y Castro. Estos emplean el «pie» para dimensionar la caja, los radios de trazado de los arcos de embocadura y el espesor de las bóvedas, mientras que el reparto de los peldaños se realiza en «palmos». Las medidas obtenidas sugieren el uso del pie de 30,2 cm y el palmo de 22,65 cm, las dimensiones usuales en Valencia durante el siglo XVI.

${ }^{8}$ El término «capialzado» se usó en cantería de distintas maneras. En la documentación de estas escaleras alude a aquellos tramos abovedados cuya curva de embocadura frontal se encuentra más elevada que la posterior. Asimismo, se llama «capialzo» a la pendiente o derrame del intradós de dicha bóveda.

9 Algunos autores asocian «engauchido» con «alabeado», siguiendo a Philibert de L’Orme (1567). Pero quizás cabría plantear otras acepciones. En Valencia, la profesora Gómez-Ferrer (2005) ha propuesto alguna alternativa ligada a términos locales. En esta variante de escalera cabría asociarlo con el término valenciano «gauxa», es decir, «brote». Aquí quizás podría aludir al brote (en la vista del alzado) de los dos arcos de embocadura de cada tramo desde un mismo origen.
} 
circunferencia y la superficie del intradós es alabeada. La unión entre ellos se resuelve también por arista. El quinto tramo sostiene una naya o galería volada, muy característica de las escaleras de honor valencianas. Su encuentro con el tramo anterior se realiza "en redó», es decir, a modo de bóveda vaída. Finalmente, la última tirada, la más tardía, descansa también sobre una bóveda capialzada aunque claramente diferenciada del resto por su heterodoxo trazado y pronunciada pendiente.

Siguiendo la tradición valenciana, el antepecho o «apitrador» apoya sobre una «copada y bocell segons art de piquer», es decir, sobre una moldura decorativa que remata los planos volados de huella y tabica en la testa del escalón para no restarle amplitud. Joseph Gelabert (1653) la llamaba «escursinat», siendo habitual en las escaleras de la Corona de Aragón. Dicho antepecho está resuelto con grandes losas de piedra sin caladuras y posee apilastrados en el inicio y final de todos los tramos. Años después, el maestro Guillem del Rey coronó estas agujas con bolas dando a la escalera un aire clasicista.

Existen dos importantes diferencias frente a otros ejemplos andaluces y castellanos. Aquí, como en otras muchas escaleras del siglo XV en la corona aragonesa, los escalones no asientan sobre la bóveda, sino que encajan en ella. El trasdós de los tizones, de un pie de grosor, fue recortado para facilitar el apoyo a montacaballo de las gradas, formadas por losas de gran tamaño que «entran en la misma bóveda». Gelabert defiende que así se descarga la estructura.

Además, respetando la tradición medieval valenciana ${ }^{10}$, las dovelas de sus vueltas se aparejan por hiladas transversales a los muros de cierre, sobre los que se derrama la escalera, cuando en otras regiones suelen ser paralelas a dichos paramentos. Esta segunda opción, recogida por Vandelvira (ca. 1578-1589), ofrece mayores ventajas de talla al concebirse la bóveda como una sucesión de arcos paralelos que facilita la traza y el corte de los lechos transversales y de las caras del intradós de los sillares. Así, la curvatura del capialzo puede simplificarse a una forma poligonal y la compleja superficie alabeada del intradós puede tallarse como una superficie reglada plana. Una licencia que resulta aún más imperceptible si se rehúnden o biselan las juntas de los sillares, como en la conocida escalera de la Real Chancillería de Granada.

En la escalera de Figuerola, sin embargo, la operación de talla es mucho más compleja. Como sus hiladas son transversales a los muros de cierre, el intradós de las dovelas adquiere forma alabeada no existiendo dos hiladas iguales. Además, aunque sus arcos de embocadura tienen idéntico radio de trazado, su distinta inclinación provoca el alabeo de la superficie de los lechos porque las juntas de testa de uno y otro frente no convergen a un mismo punto. Para salvar esta dificultad parece que se optó por reorientar la dirección de las juntas de una de las testas, evitando así el alabeo de la superficie del lecho (figura 2).

\section{REFERENTES TÉCNICOS}

Partiendo de las evidencias expuestas por la profesora Gómez-Ferrer resulta sencillo constatar que el verdadero mérito de esta escalera consistió en incorporar simultáneamente, con mayor elegancia y armonía que en ninguna otra, los logros de sus antecesoras tardogóticas: la galería, el capialzado, el engauchido y los encuentros por igual y por arista; así como sus estrategias de traza y labra.

En la ciudad de Valencia se conservan catorce escaleras capialzadas ${ }^{11}$ de casas nobles, pero sólo tres resuelven la unión o mezcla del intradós del tramo y la naya: el Palacio de Mercader, el de En Bou y la casa de los Juliá o de los Barones de Santa Bárbara. De ellas se deduce que, en un primer momento pudieron utilizarse los encuentros continuos entre los tramos (similares a una bóveda vaída por hiladas cuadradas) y más tarde las uniones por arista. Aunque Arturo Zaragozá (26) sugiere que los encuentros en arista entrante, enlazando hiladas con piezas en «V» invertida por la tradicional disposición transversal del aparejo de sus sillares, podrían ser contemporáneos a los anteriores.

En contra de lo que sugiere su lectura estrictamente compositiva, los ejemplares del colegio de Orihuela y del Corpus Christi combinan este amplio repertorio de soluciones técnicas con mayor sofisticación que la escalera imperial de San Miguel de los Reyes. Quizás pudo darse algún intercambio de conocimientos entre Figuerola y Joan Anglés. En 1592 el primero trabajó a las órdenes del segundo en el Puente del Mar de Valencia, cuatro años después de concluir la escalera de Orihuela.

\section{ACOTACIÓN DE LA INVESTIGACIÓN}

Esta investigación pretende paliar en parte el déficit señalado poniendo en relación la escalera moderna más relevante del antiguo reino de Valencia con sus predecesoras tardogóticas. A tal fin, se ha determinado de manera empírica su posible traza con una triple finalidad: intentar acreditar la pervivencia de los métodos tardomedievales de trazado y control de la forma (27) de las escaleras valencianas de patio en el proyecto y la construcción de los ejemplares de la Edad Moderna; establecer el grado de coincidencia de estas hipótesis con las propuestas en los manuscritos de cantería; para, en último término, calibrar unas pautas metodológicas útiles para el estudio de otros ejemplares coetáneos.

Se realizó una medición muy precisa de la escalera con un escáner láser $3 \mathrm{D}^{12}$. Se descartó el mallado tridimensional de los puntos, operando directamente sobre ellos. Las trazas se analizaron en dos dimensiones, siguiendo a los antiguos maestros. Al efecto, se extrajeron planos de

\footnotetext{
${ }^{10}$ Las bóvedas valencianas medievales y modernas solían aparejarse por hiladas transversales, con independencia de su naturaleza y dimensiones.

${ }^{11}$ Casa de los Selva, palacio de Scala, Barones Catalanes de Vallvert, Malferit, Mercader, Queixals, Barón de Santa Bárbara, En Ripoll, En Bou, Barón de Vallvert, Valeriola, Escrivá y Boil, Catalá de Valeriola y casa en la calle Baja no 48.

12 Se usó un modelo Faro Scan 130 y se realizaron siete estacionamientos, ajustados para tomar un punto cada 2 mm. El máximo error de enlace fue de $5 \mathrm{~mm}$. Se empleó la aplicación «Scene» versión 5 para el tratamiento de la nube de puntos.
} 


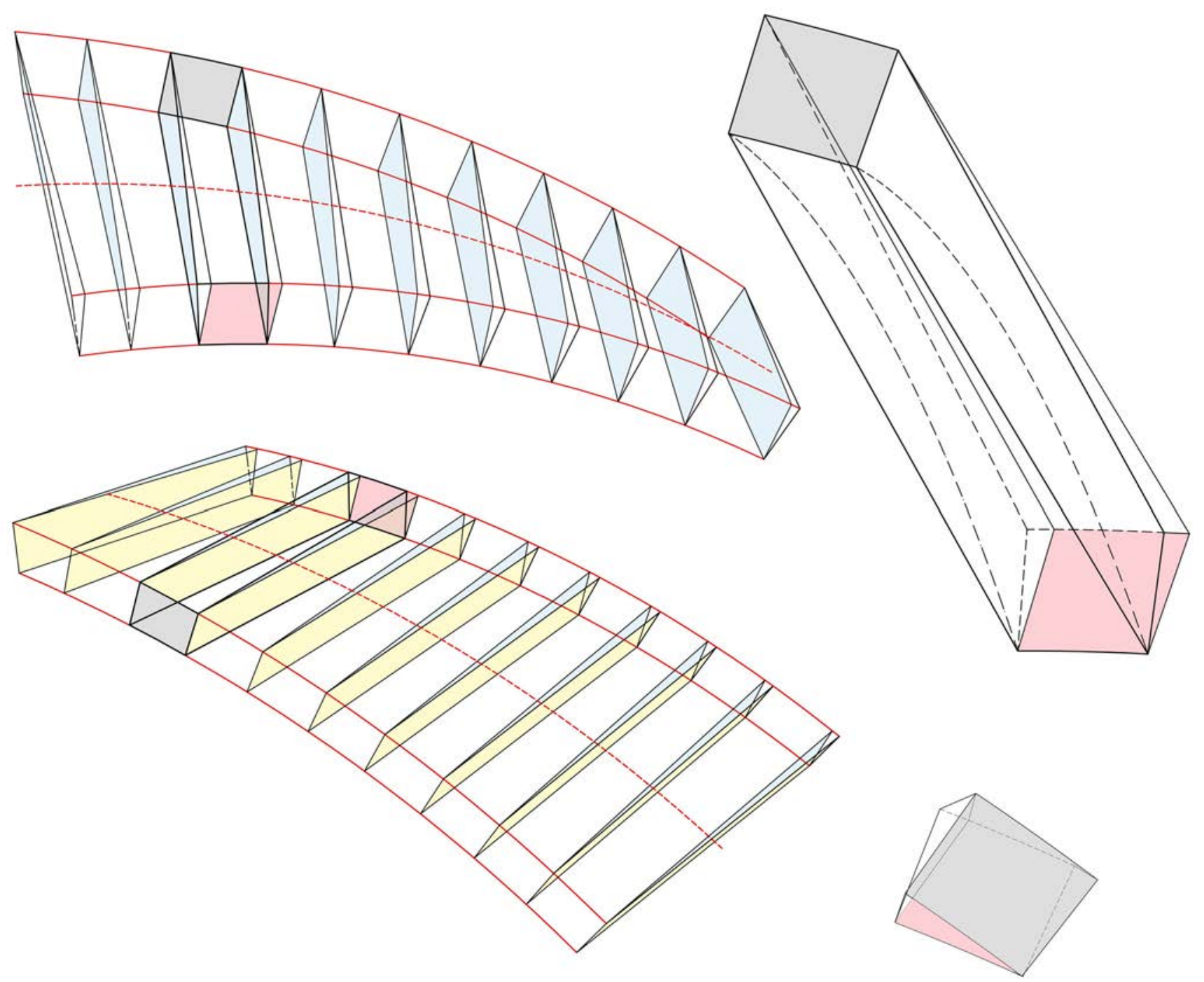

Figura 2. Posible corrección de las superficies de los lechos en las escaleras capialzadas modernas valencianas para evitar su alabeo (planos amarillos). Se redireccionan las juntas de la testa posterior (rosa), disponiéndolas en paralelo a las de la testa frontal (gris).

corte de la nube de puntos mediante la aplicación «Escuadra», implementada por el profesor Adolfo Alonso Durá. Este software no comercial aísla los puntos más próximos a un plano virtual dado permitiendo probar en AutoCAD sencillos trazados geométricos sobre aquellos puntos de la nube contenidos en cada plano de corte. Así, se ha podido analizar la forma actual de sus arcos, evaluar las deformaciones, los desplomes y el despiece exacto de sus sillares. A partir de estas se ha confeccionado una hipótesis de traza de sus curvas directrices, comparando los resultados con los tratados y otras monteas conocidas de las escaleras tardogóticas de honor.

Se han considerado las trazas propuestas por Alonso de Vandelvira (1578-1589), Juan de Portor y Castro ${ }^{13}$ (1708) y Thomás Vicente Tosca (1727), los únicos autores hispanos que abordan esta variante de escalera, aunque los dos últimos en fechas muy posteriores a su construcción (figura 3). El padre Tosca, además, sólo alude escuetamente a esta traza en la proposición VIII de su tratado XV «De la Montea y Cortes de Cantería», cuyo problema titula «formar una escalera con vueltas que formen arista». También han resultado útiles las enseñanzas de Joseph Gelabert (1653), quien aporta varios antecedentes tipológicos.

\section{CRITERIOS DE ANÁLISIS}

Se ha elaborado una hipótesis gráfica de trazado para cada tramo, pero considerando que pudiera existir una pauta común para todos ellos. En cada tirada se analizaron sus dos secciones longitudinales extremas, es decir, los arcos de embocadura o curvas directrices de los frentes del hueco y del muro que algunos maestros llaman «ydas». A continuación, se obtuvo el capialzo, esto es, la curvatura o derrame hacia el paramento de las secciones transversales del intradós, también llamadas «vueltas». Esta definición de las curvas directrices de todas las aristas de cada sillar permite su talla directa con ayuda de una simple regla y también evaluar la singularidad o estandarización de cada pieza dentro del conjunto.

${ }^{13}$ El manuscrito de Portor profundiza más que el resto en las escaleras claustrales. Incluye siete variantes clasificadas en dos tipos, por la disposición de sus juntas. Además, sus minuciosos gráficos resultan de gran interés. 

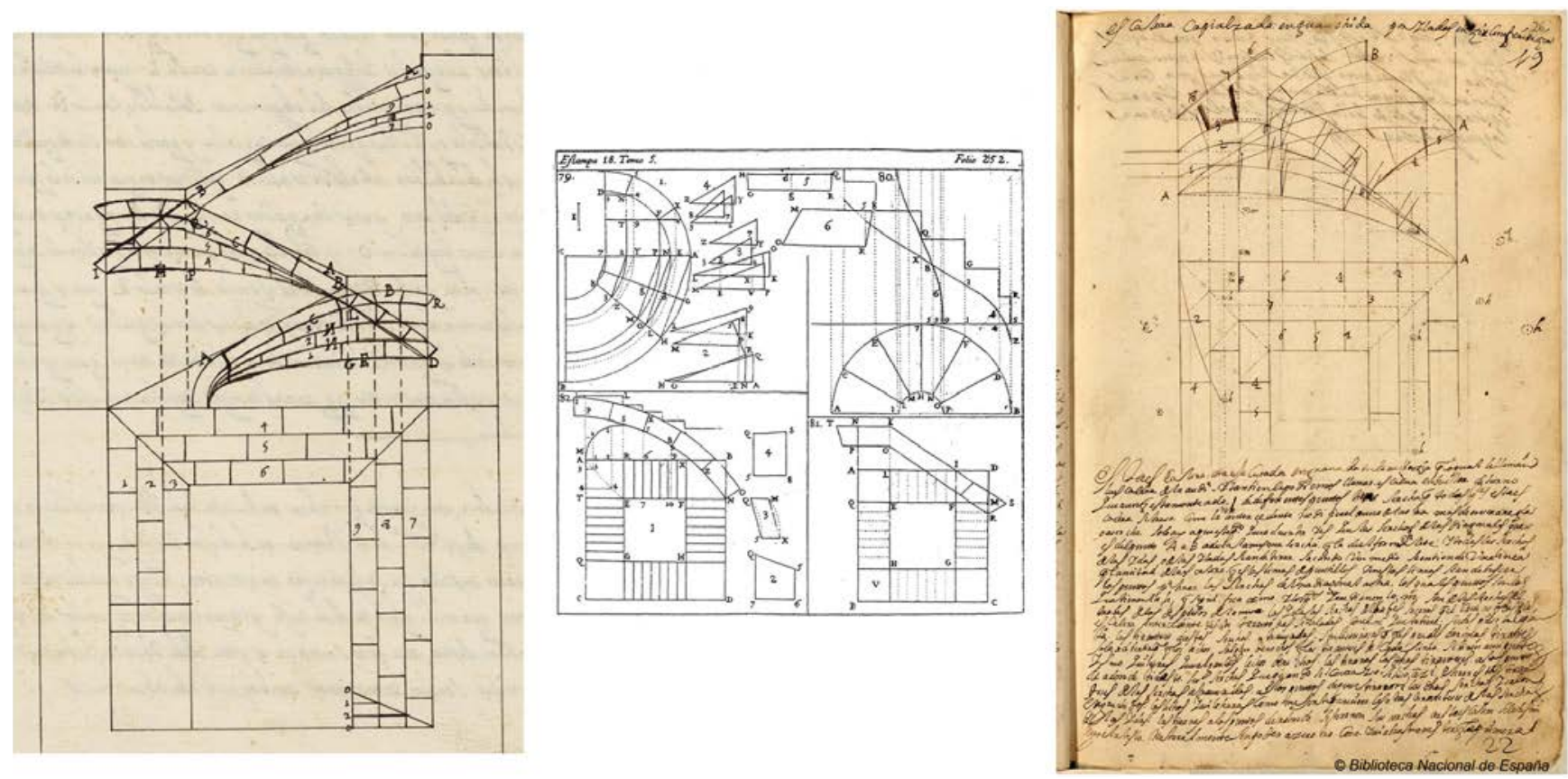

Figura 3. Propuestas de traza de esta variante de escalera en los manuscritos de cantería. A la izquierda, la solución de Alonso de Vandelvira (ca. 1585); copia de Felipe Lázaro de Goiti (1646), fol. 102. En el centro, traza de Thomás Vicente Tosca (1727), tomo 5, estampa 18, fol. 252. A la derecha, traza de la «escalera capialzada, engauchida por yladas en zircunferenzia» de Juan Portor y Castro (1708), fol. 19.

Aunque en el mercado existen aplicaciones que proporcionan los radios de curvatura más próximos a un conjunto finito de puntos aquí se ha hecho por tanteo juicioso, respetando las directrices de los tratados. Este criterio permite ponderar mejor los errores e imprecisiones de diseño, talla, montaje y deformación en servicio de la bóveda. Dichos trabajos, no obstante, acarrean incertidumbres y, para evaluar su fiabilidad, debe considerarse el pensamiento técnico de los antiguos maestros y sus semejanzas con otros ejemplos análogos. También se han obtenido las equivalencias en pies valencianos de las medidas consideradas, como una posible garantía adicional de fiabilidad.

El proceso de análisis seguido se adapta a lo señalado por los tratadistas para estas escaleras voladas de caja cuadrada. Vandelvira ${ }^{14}$ puntualiza que debe obtenerse primero el radio del trasdós de la curva directriz del hueco de la escalera. La traza comienza por el arranque para ir enlazando los arcos de cada tramo con sus contiguos en las charnelas de los rellanos «de tal manera correspondan las unas cerchas de la primera vuelta con las de la segunda [...] que parezca ser toda una voveda». Idéntico criterio sigue Portor, quien también advierte de la necesidad de combinar las «serchas» (arcos de circunferencia) de las distintas tiradas. El Padre Tosca, además, establece que los arcos de embocadura deben tener un palmo o un pie de canto, una regla seguida por los ejemplos valencianos investigados.

Lamentablemente, ninguno ofrece una regla para obtener los radios, ni dicen cómo fijar los centros de trazado. Juan de Portor los señala en su montea y Tosca sugiere una posición y un radio aproximados para la curva directriz del intradós o «mocheta del claro», y lo hace condicionando su paso por el punto preestablecido de encuentro con el siguiente tramo, si bien deja abierta su elección "à prudencia del architecto». Solo Vandelvira explica con un mínimo detalle la obtención de la embocadura del capialzado en su encuentro con el muro. No obstante, todos ellos establecen las condiciones que debe cumplir este arco: la curva directriz de «yda» o «cercha adulçada», en palabras de Vandelvira, debe ser tangente a la línea auxiliar («a trainel») que une los «dientes» de las huellas y tabicas de los peldaños o, al menos, intentar «que se allege lo mas que pudiere a la dicha línea». Como se ha señalado, además hay que forzar su paso por un punto preestablecido en las charnelas de los tramos para que todas las bóvedas aparenten tener continuidad.

Algo similar ocurre con el trazado de la arista del rellano. Solo Juan de Portor se detiene en ello, señalando que «las serchas de las diagonales que el del punto A a B a ser la mesma sercha q la del formalete», es decir, el semiarco de esquina bajo el rellano debe trazarse con el mismo radio que el arco de embocadura.

Los manuscritos no explican la traza de los cortes transversales del capialzado, tan característicos en Valencia. Valdelvira y Portor proponen aparejos longitudinales y Tosca omite muchos aspectos en su esquemática traza.

\section{LA ESCALERA DEL CORPUS CHRISTI: HIPÓTESIS DE TRAZA}

Aunque existen diferencias en las propuestas de cada tratadista, todos respetan unas pautas comunes: optan por montear primero las parejas de arcos de las «ydas», es decir, las embocaduras o testas del hueco de la escalera y de la unión

\footnotetext{
${ }_{14}$ Las dos copias conocidas del manuscrito de Vandelvira proporcionan una descripción muy similar, con ligeros matices poco trascendentes en cuanto a las ideas generales aquí expuestas.
} 
con el muro lateral. A continuación, se trazan las aristas, si se opta por este encuentro bajo las mesetas. Finalmente, se dibujan las curvas de las «vueltas», es decir, los derrames del capialzo. Así obtienen las plantillas de todas las aristas de cada sillar.

Para determinar el posible método de trabajo de Figuerola se han seguido ordenadamente los pasos anteriores, dibujando directamente sobre las secciones de la nube de puntos y operando de forma empírica sobre aquellas cuando los tratados omitían algún procedimiento intermedio en la secuencia gráfica.

En primer lugar, según la costumbre, se replantearon las gradas y el plano de los rellanos sobre los muros de la caja trazando, a continuación, la línea auxiliar que une sus «dientes», como propone Vandelvira. Siguiendo a Juan de Portor y Castro se trazaron primero las curvas directrices de las testas voladas comenzando por el tramo de arranque. Tosca llama a esta curva «mocheta del claro» y Vandelvira «cercha adulçida».

A continuación, se hallaron los posibles centros y radios de curvatura de estos arcos del hueco o de testa sobre la nube de puntos. Se comprobó que la curva resultante en cada tramo era tangente a una línea auxiliar paralela a la pen- diente de las gradas previamente trazada. Ésta guarda con aquella una distancia de un pie valenciano $(30,2 \mathrm{~cm})$, que es precisamente el grosor de la bóveda, según Tosca. Sólo faltaba por determinar la estrategia geométrica seguida por Figuerola para obtener la posición del centro de trazado de la curva directriz y su radio. Este arco debe cumplir la triple condición de ser tangente al segmento anterior, tener un radio conocido y pasar por el punto de encuentro con la curva del tramo inferior.

En el segundo tramo este punto es conocido y se encuentra en la línea del pavimento. En los restantes tramos se obtiene de forma inmediata si se resuelven en orden ascensorial, ya que es el punto de unión con el arco de vuelo del tramo inmediatamente inferior. Por tanto, basta con trazar un arco de circunferencia desde dicho punto con un radio conocido que corte al segmento paralelo a la línea de pendiente, dispuesto a una distancia igual al citado radio. El punto de corte obtenido será el centro de la curva directriz del arco de «yda» (figura 4).

Se observó que el radio de estas curvas directrices de «yda» tiene casi siempre un valor igual o muy próximo a la distancia entre los muros que delimitan el tramo en cuestión. Por tanto, resulta muy sencillo establecer la posición de su cen-
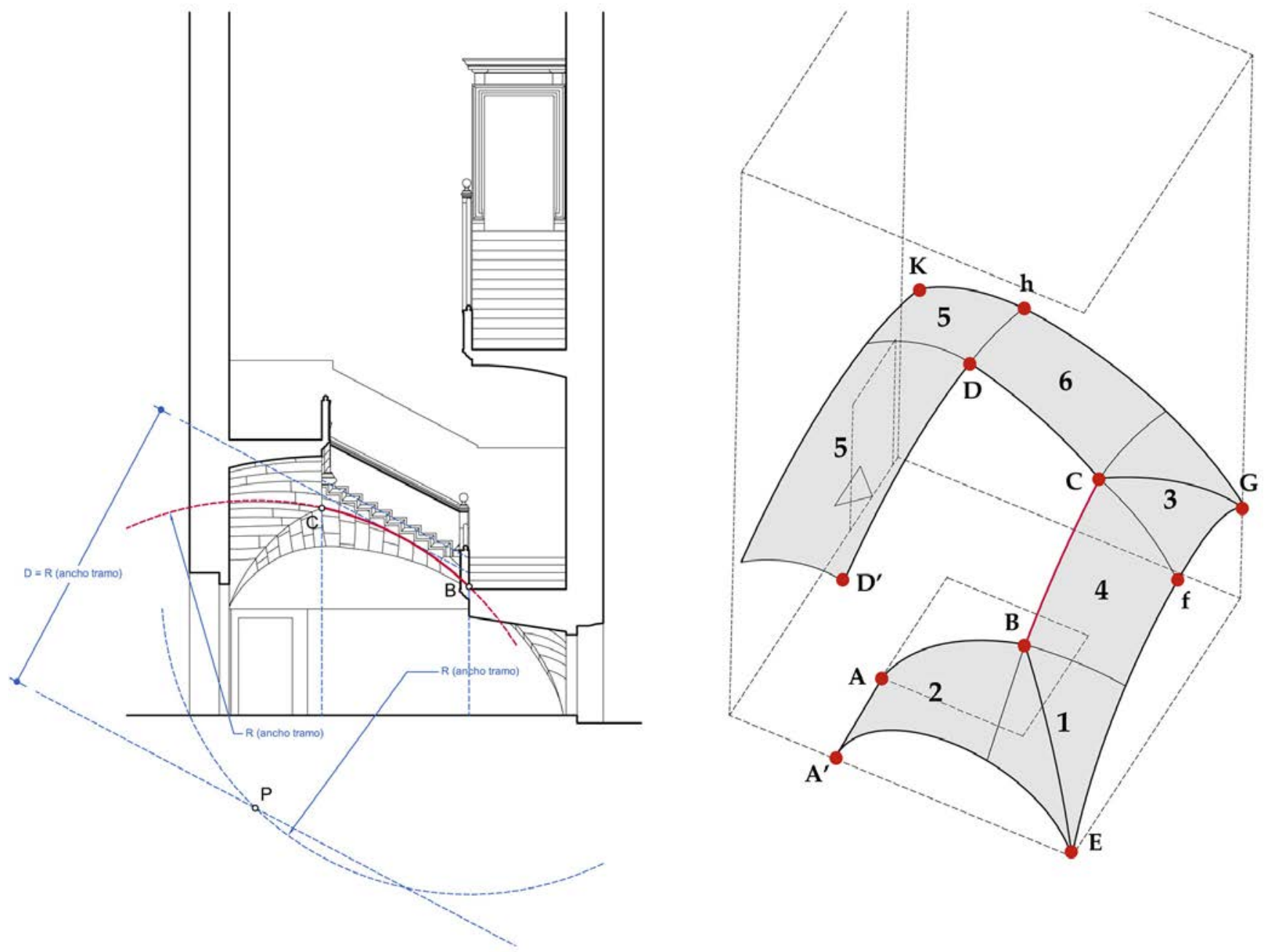

Figura 4. Procedimiento válido para determinar los centros de las curvas de embocadura de los capialzados de la escalera cuando se conoce el radio del arco, un punto de paso y una recta tangente a ellas (izquierda). Definición de las superficies del intradós de la escalera a partir de las curvas directrices previamente trazadas y posible secuencia constructiva de los tramos que delimitan dichas curvas directrices (derecha). 

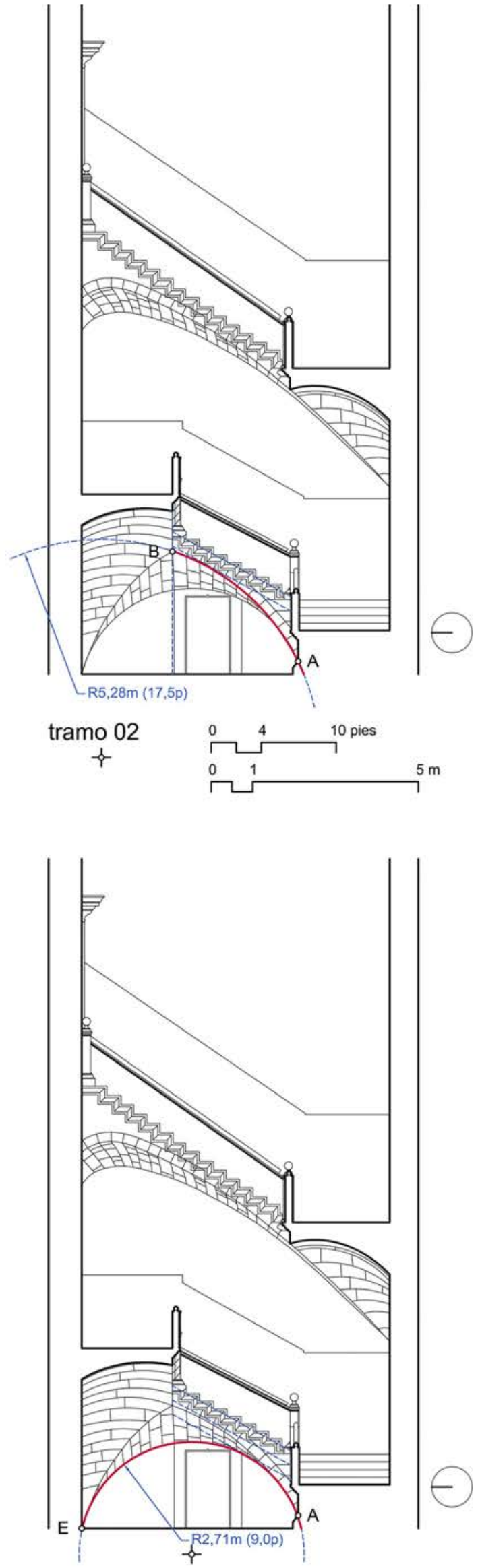

tramo 02

Figura 5. Hipótesis de traza de las curvas directrices de los arcos de embocadura del segundo tramo de la escalera del Patriarca. tro de trazado respetando las tres condiciones anteriormente descritas. Se constata que en el segundo tramo - que es, en realidad, el primero volado- esta distancia es de 17,3 pies y el radio del arco ronda los 17,5 pies. En el tercer tramo la distancia entre los muros es de 26 pies y el radio de su arco exterior tiene unos 20 pies. Por último, el ancho del cuarto tramo es de 24,5 pies, magnitud muy próxima también al radio de curvatura del arco de borde de dicha tirada. La excepción manifestada en el tercer tramo podría deberse a la necesidad de forzar el encuentro con los contiguos mediante una arista entrante (figuras 5 y 6 ).

La coincidencia del radio con el ancho de la caja acaso pretendería reducir el gasto de piedra construyendo bóvedas muy tendidas sin mermar su fiabilidad estructural. Como su replanteo se hacía en la propia estancia, quizás adoptasen el mayor radio que cabía dibujar en el recinto. Y este solía corresponderse precisamente con la distancia entre los apoyos, una dimensión fácil de recordar (tabla 1).

Dicha regla se usó antes en las escaleras tardogóticas valencianas. La mayoría tenían bóvedas capialzadas de un único tramo que transmitía sus esfuerzos a los muros extremos. Se ha comprobado que en el palacio de Vallvert y en el de Scala el radio mayor y la distancia entre apoyos se aproximan a los 34 pies. Y también en el palacio de los Catalá de Valeriola, si se tiene en cuenta que allí la bóveda continúa por el interior de la «civadera» (almacén de cebada) dispuesta en el hueco de la escalera.

Idéntica pauta parecen seguir las tres escaleras de honor de la primera mitad del siglo XVI conservadas en la ciudad de Valencia que mezclan por igual en su esquina las bóvedas del tramo y de la galería. En el Palacio de Mercader ambas medidas son de 32 pies. Además, el intradós de su bóveda capialzada guarda una distancia mínima de un pie con la línea de pendiente de los peldaños, como también ocurre en la escalera del Corpus Christi. En el Palacio de En Bou es de 11 palmos -aunque aquí la distancia entre apoyos fue obtenida por métodos indirectos- y la bóveda tiene solo un palmo de canto. Esta última es la única modulada en palmos, como sugiere Tosca. Ello aún reduce más el gasto de piedra.

Asimismo, en estos ejemplos previos, para facilitar el encuentro «por ygual» (redondeado) entre la bóveda del tramo y la de la naya parece que también se hace coincidir el radio del arco de la galería con la distancia entre sus apoyos. Al menos eso apuntan los ejemplares de los palacios de Mercader y de En Bou. Como se ha señalado, siglos más tarde, Juan de Portor recomendaba una solución equivalente para el radio de las aristas.

Conocido el proceso para la obtención de los arcos del vuelo se procedió a analizar el trazado de las curvas directrices de encuentro de la bóveda capialzada con los muros de cierre de la escalera. El segundo tramo es el más dispar por el deseo del proyectista de definir mediante una sola curva un capialzado recto. Este propicia a su vez la formación de una poderosa arista en la esquina, para un mejor apeo de la escalera, y la apertura de un hueco de paso bajo la bóveda. Esta curva tiene unos 9 pies de radio, aproximadamente la mitad del usado para la testa del vuelo.

Como novedad, a partir del tercer tramo la curva directriz del muro pasa a estar formada por dos ramas de arco. La de mayor 


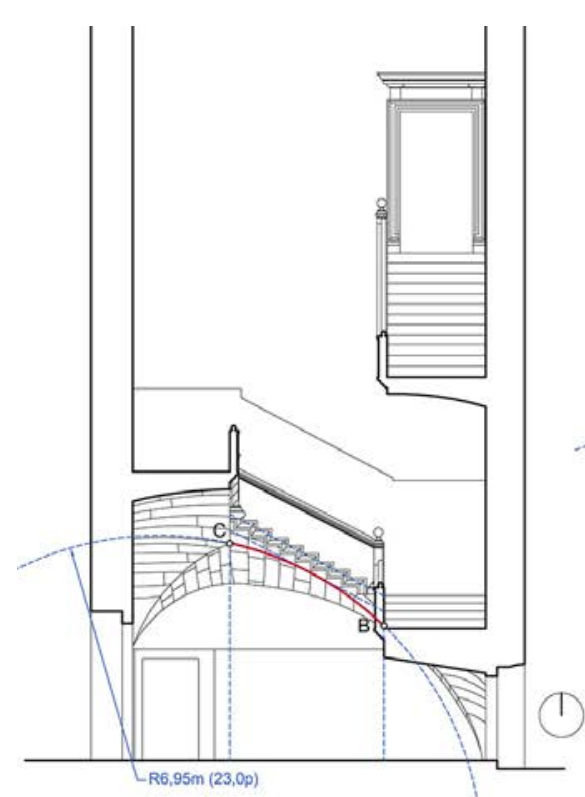

tramo 03

$+$

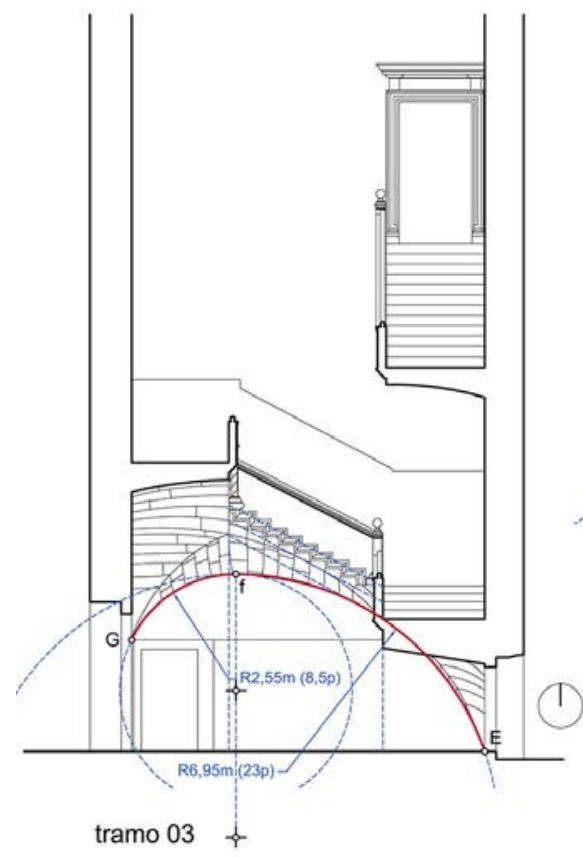

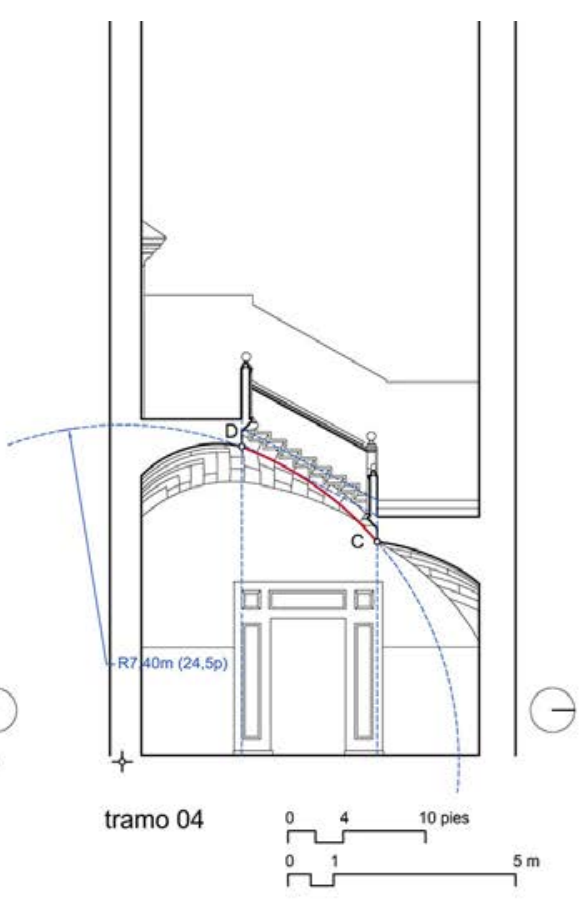

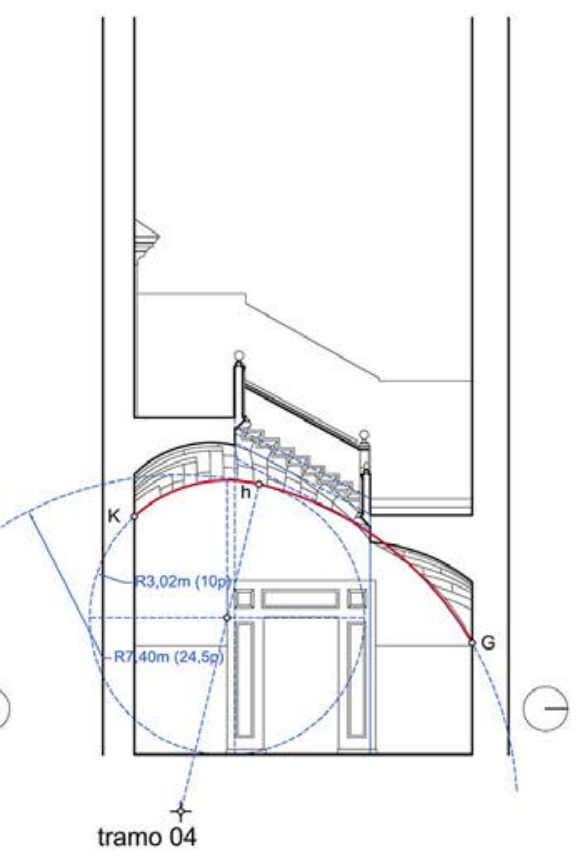

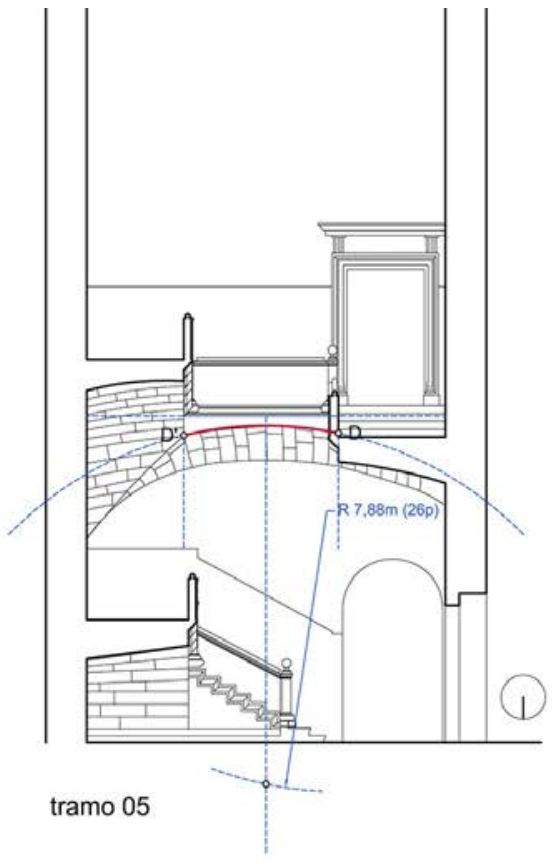

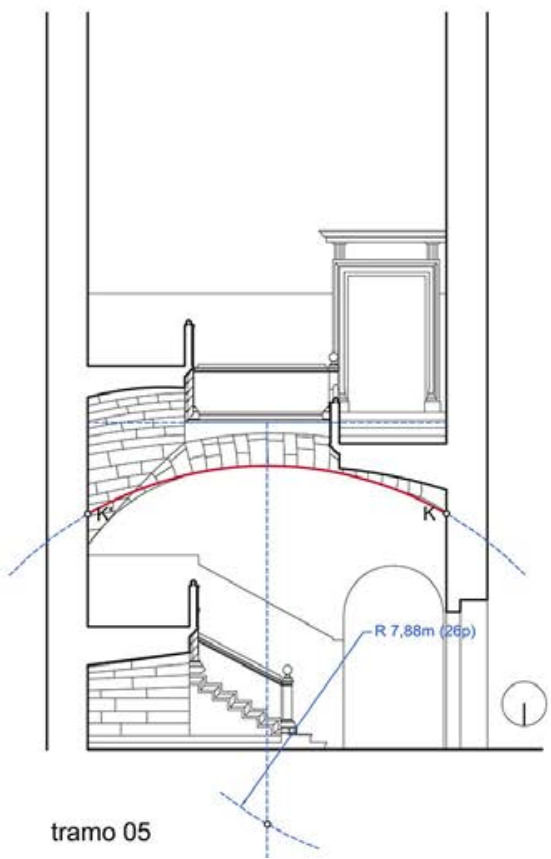

Figura 6. Hipótesis de trazado de las curvas directrices de los arcos de embocadura de los tramos tercero, cuarto y quinto de la escalera del Patriarca.

radio sigue un criterio similar al de sus homólogas del frente volado: en este caso también se predetermina un radio, se conoce un punto por el que debe pasar el arco y es tangente a una recta dada. Se ha comprobado que el radio es el mismo que el utilizado para el arco de vuelo, es decir, el ancho de la caja: unos 20 pies en el tercer tramo y 24,5 pies en el cuarto. El punto de trazado conocido ha sido denominado con las letras A, B, C y D en las figuras 5 y 6 . En este caso la recta tangente es una paralela a la línea de pendiente del tramo, trazada a 2 pies de aquella.

Por su parte, las ramas de menor radio de las curvas directrices de los tramos tercero y cuarto fueron trazadas también con radios preestablecidos de unos 8,5 y 10 pies, respectiva- mente. En este caso, debió hacerse algún ajuste en la traza. Los puntos de tangencia entre ambas curvas, denominados «f» $\mathrm{y}$ «h» en la figura 6, se alejan de la línea o charnela de encuentro entre los tramos. Ambos parecen ser un resultado del proceso y no un dato de partida.

La construcción geométrica de la curva directriz del cuarto tramo interior merece una especial atención. Su arco menor, que define la sección transversal de la galería, parece ser tangente al plano del pavimento y su centro se sitúa próximo al vértice inferior izquierdo del dintel de la monumental portada pétrea. Este hecho no parece casual, más bien denota una voluntad compositiva. Además, la curva directriz de la naya decae hacia el hueco de la escalera, de 
Tabla 1. Comparativa entre la dimensión de los tramos y los posibles radios de trazado.

\begin{tabular}{|l|l|l|l|}
\hline & \multicolumn{1}{|c|}{ Largo tramo } & \multicolumn{1}{c|}{ Curva directriz hueco } & \multicolumn{1}{c|}{ Curva directriz muro } \\
\hline Tramo 01 & 24,5 pies & macizo & macizo \\
\hline Tramo 02 & 17,3 pies $^{*}$ & 17,5 pies & 9,0 pies \\
\hline Tramo 03 & 26,0 pies & 20,0 pies & 20,0 pies \\
\hline Tramo 04 & 24,5 pies & 24,5 pies & 24,5 pies \\
\hline Tramo 05 & 26,0 pies & 26,0 (galería) & 26,0 (galería) \\
\hline
\end{tabular}

* Distancia libre, medida desde el macizo de arranque de la escalera.

manera que el punto «h» de tangencia entre ambas ramas se aleja de la vertical y, por tanto, la línea que une ambos centros también se inclina.

Llegados a este punto, sólo quedan por conocer los radios de las aristas y el procedimiento seguido para determinar la curvatura de las juntas transversales de las dovelas. Los primeros fueron obtenidos trazando una sección vertical de la nube de puntos por el plano de cada arista. Ambas aristas tienen un radio de unos 17,5 pies, el mismo que arroja la curva directriz del frente volado del segundo tramo.

La determinación de las juntas de lecho del segundo tramo es inmediata por tratarse de juntas rectas, pero no así en los sucesivos, que poseen una pronunciada curvatura. Se observa que, para su determinación, se usó un procedimiento similar a los descritos por Vandelvira y Portor. Se tendió una curva directriz auxiliar contenida en un virtual plano vertical, paralelo y equidistante de las dos embocaduras. Este arco, que proporciona la altura de la junta en el centro del tramo, arroja un radio de unos 20 pies en el tercer tramo y de 24,5 pies en el siguiente. Por tanto, es igual al de los arcos de embocadura del tramo. La construcción geométrica que determina su posición en la traza es la misma de las testas, pero situando aquí el segmento tangente a la curva a un pie y medio de distancia de los «dientes». La intersección de dicho plano virtual con la curva de la arista del rellano define el punto preestablecido de arranque del arco.

Para obtener las cotas de los tres puntos de paso de la curva inferior definitoria de las aristas de los sillares debían trazarse tres verticales desde la proyección en planta de la
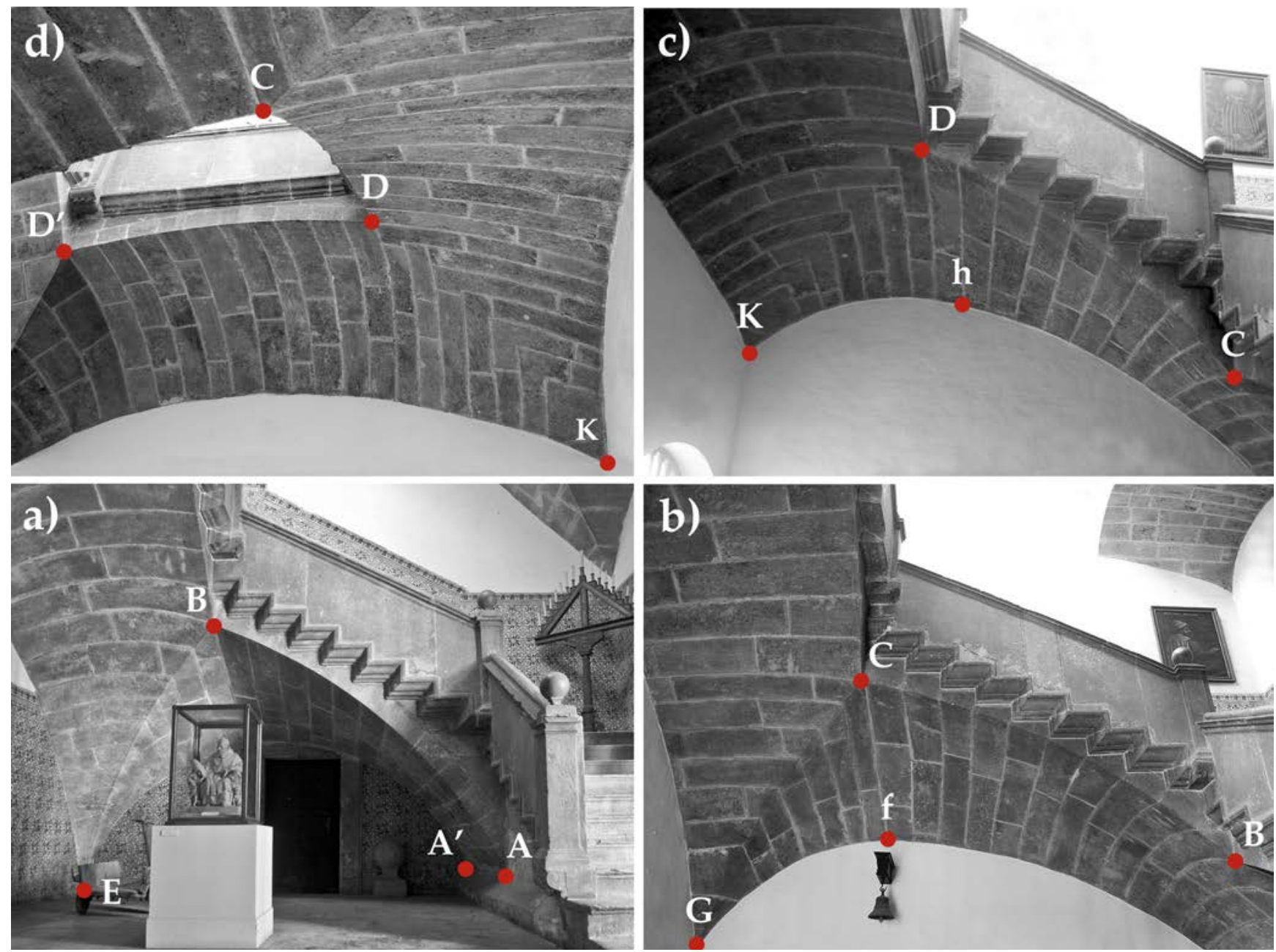

Figura 7. Vistas cenitales de los cinco tramos abovedados analizados con indicación de los posibles puntos de conexión de las curvas directrices. 
junta del lecho buscando su intersección con los tres arcos de «yda».

\section{CONCLUSIONES}

La escalera del Corpus Christi de Valencia respeta en esencia los confusos criterios prescritos por los tratadistas. Pero contrariamente a aquellos, que dejan muchos flecos abiertos, Francisco Figuerola parece desplegar unas operaciones de traza muy concretas para obtener las curvas directrices. $\mathrm{El}$ análisis gráfico del elemento sugiere que dicho arquitecto podría haber sorteado hábilmente un complejo problema de geometría plana fijando como datos de partida algunas variables de difícil determinación.

No obstante, aunque esa simplificación formal resuelve en parte la dificultad intrínseca que conlleva la definición de tan complejos volúmenes, la labra de sus piezas exige una considerable pericia. De hecho, habría que suponer el uso de modelos previos a escala para valorar los resultados e introducir ajustes.
Por tanto, el control de la forma de esta escalera respeta las estrategias de traza de tradición medieval. Se apoya en curvas directrices -es decir, líneas y no planos-, para definir las superficies de doble curvatura, generalmente no esféricas. De hecho, cuando se observa detenidamente, no es difícil detectar a simple vista que, al igual que sus antecesoras, la escalera está formada por varios elementos construidos por fases y sutilmente enlazados para aparentar que forman un conjunto unitario (figura 7).

Francisco Figuerola aunó, en una misma estructura, una gran diversidad de recursos técnicos que no habían sido empleados de manera conjunta hasta entonces para dar respuesta a un problema que tampoco había sido planteado hasta la fecha en esos términos. La voluntad de lograr un diseño armonioso, aparentemente ligero, técnicamente complejo y estructuralmente estable -que, además, debía salvar ciertos condicionantes funcionales como la integración de los tres accesos-, desembocó en esta innovadora propuesta.

\section{REFERENCIAS}

(1) Bérchez Gómez, J. y Gómez-Ferrer Lozano, M. (2013). El Real Colegio de Corpus Christi o del Patriarca de Valencia desde el espejo de la arquitectura. En Una Religiosa Urbanidad. San Juan de Ribera y el Colegio del Patriarca en la cultura artística de su tiempo (pp. 29-49). Valencia: Real Academia de BB.AA. de San Carlos.

(2) Íñiguez Almech, F. (1952). Casas Reales y Jardines de Felipe II, p. 103, Madrid, Consejo Superior de Investigaciones Científicas.

(3) Gelabert, J. (1653). Edición de Rabasa Díaz, Enrique. (2011). Vertaderas traçes del Art de picapedrer, pp. 324-349, Mallorca: Col-legi Oficial d'Arquitectes de les Illes Balears.

(4) Fornés y Gurrea, M. (1857). Observaciones sobre la práctica del arte de edificar, $2^{\mathrm{a}}$ edición, pp. 8-9 lám. II y III, Valencia: Imprenta de Cabrerizo.

(5) Vandelvira, A. (1646). Fragmentos de dibuxos y discursos de arquitectura [manuscrito] de Alonso de Vande Elvira; copiados [tachado] ordenados acaso por Felipe Lázaro de Goiti, fol. 99-103, Madrid: Biblioteca Nacional. Mss. 12.719.

(6) Vandelvira, A. (s. XVII). Exposición y declaración sobre el tratado de Cortes de Fabricas que escriuio [Alonso] de Valdevira [manuscrito] / por... [Bartolomé] de Sombigo y Salcedo, Madrid: ETS de Arquitectura. Sign. Raros 31. (Versión digitalizada: Colección Digital de la UPM, http://cdp.upm.es/)

(7) Portor y Castro, J. 1708. Cuaderno de Arquitectura (manuscrito), fol. 19v-19r, Madrid: Biblioteca Nacional. Mss. 9114.

(8) Piqueras García, R. (2006). Francisco Figuerola, “Maestro de Cantería” entre los siglos XVI y XVII. De Mora D’Ebre (Tarragona) a Almansa (Albacete). Al-Basit, 50: 177-200.

(9) Zaragozá Catalán, A. (2013) Las escaleras con bóveda continua de piedra: técnicas y significados. El episodio valenciano de la edad moderna. En Antista, G. y Bares, M. (Eds.), Le scale in pietra a vista nel Mediterraneo (pp. 21-36). Palermo: Edizioni Caracol.

(10) Barbé Coquelín de Lisle, G. (1976). El Tratado de Arquitectura de Vandelvira y la estereotomía en España. En Actas del XXIII Congreso Internacional de Historia del Arte (v. II, pp. 226-232). Granada: Universidad de Granada.

(11) Palacios Gonzalo, J. C. (1984). Intervención y convención en las técnicas constructivas del renacimiento español (estereotomía renacentista del tratado de Vandevira). (Tesis doctoral). Madrid: Universidad Politécnica de Madrid.

(12) Salcedo Galera, M. (2017). Construcción pétrea en la Granada del Renacimiento. (Tesis doctoral). Cartagena: Universidad Politécnica de Cartagena.

(13) Zaragozá Catalán, A., Calvo López, J. y Natividad Vivó, P. (2012). Stereotomic Exchanges between Iberia and France in the 16th Century: Benoît Augier, Valencian Stairways and the Escalier de Toulouse. En Nuts \& Bolts of Construction History: Culture, Technology and Society: Proceedings of the Fourth International Congress on Construction History, Paris, 3-7 July 2012 (v.1, pp. 385-392). Paris: Picard.

(14) Gómez-Ferrer Lozano, M. (2005). Patios y escaleras en los palacios valencianos del siglo XV. En Historia de la Ciudad IV (pp. 113-142). Valencia: Colegio Territorial de Arquitectos.

(15) Zaragozá Catalán, A. (2010). Cuando la arista gobierna el aparejo: bóvedas aristadas. En Arquitectura en construcción en Europa en época medieval y moderna, (pp.187-224). Valencia: Universitat de València.

(16) Falomir Faus, M. (1996). Arte en Valencia, 1472-1522, p. 201, Valencia: Consell Valencià de Cultura.

(17) Gelabert (1653). Edición de Rabasa Díaz, Enrique. (2011). Vertaderas traçes del Art de picapedrer, pp. 338, Mallorca: Col-legi Oficial d'Arquitectes de les Illes Balears.

(18) Vandelvira (1646). Fragmentos de dibuxos y discursos de arquitectura [manuscrito] de Alonso de Vande Elvira; copiados [tachado] ordenados acaso por Felipe Lázaro de Goiti, fol. 100, Madrid: Biblioteca Nacional. Mss. 12.719. 
(19) Tosca, T. V. (1727). Tratado de la Montea y Cortes de Cantería. Segunda impresión corregida y enmendada, Libro V. De las vueltas para Escaleras, y otros Arcos, y Bóvedas irregulares, p. 250, Prop. VIII, Madrid: Imprenta de Antonio Marín.

(20) Wethey, H. E. (1964). Escaleras del primer Renacimiento español. Archivo Español de Arte, 37 (148): $295-306$.

(21) Martínez Montero, J. (2014). Génesis y evolución tipológica de la escalera en la arquitectura del renacimiento en España. Bilduma Ars. Revista del Departamento de Historia del Arte y Música de la Universidad del País Vasco, 4: 7-36.

(22) Gelabert, Joseph. (1653). Edición de Rabasa Díaz, Enrique. (2011). Vertaderas traçes del Art de picapedrer, pp. 326-348, Mallorca: Col-legi Oficial d'Arquitectes de les Illes Balears.

(23) Carvajal Alcaide, R. (2011). Stairs in the Architecture Notebook of Juan de Portor y Castro: An Insight into Ruled Surfaces. Nexus Network Journal, vol. 13, Issue 3: 631-648.

(24) Gómez-Ferrer Lozano, M. (2005). Patios y escaleras en los palacios valencianos del siglo XV. En Historia de la Ciudad IV (pp. 121-134). Valencia: Colegio Territorial de Arquitectos.

(25) Benito Domenech, F. (1981). La arquitectura del Colegio del Patriarca y sus artífices, p. 76, Valencia: Federico Domenech.

(26) Zaragozá Catalán, Arturo. (2013). Las escaleras con bóveda continua de piedra: técnicas y significados. El episodio valenciano de la edad moderna. En Antista, G. y Bares, M. (Eds.), Le scale in pietra a vista nel Mediterraneo (p. 24). Palermo: Edizioni Caracol.

(27) Rabasa Díaz, E. (2013). Estereotomía: teoría y práctica, justificación y alarde. Informes de la Construcción, vol. 65 (nº extra-2): 5-20. 